Onkologe 2010 · 16:119-120

DOI 10.1007/s00761-010-1798-6

๑) Springer-Verlag 2010

\section{T. Wiegel ${ }^{1} \cdot$ P.M. Schlag ${ }^{2}$}

${ }^{1}$ Klinik für Strahlentherapie und Radioonkologie, Universitätsklinikum Ulm, Ulm

${ }^{2}$ Charité Comprehensive Cancer Center, Charité - Universitätsmedizin Berlin,

Campus Mitte, Berlin

\title{
Viel Bewegung in der interdisziplinären Therapie des Nierenzellkarzinoms
}

Mit dem Themenheft Nierenzellkarzinom geben wir Ihnen einen aktuellen Überblick zu derzeit wichtigen Aspekten in Diagnostik und Therapie dieses Tumors. Die Beiträge dieses Themenhefts sind insbesondere unter dem Blickpunkt gewählt, Schnittpunkte der interdisziplinären $\mathrm{Zu}$ sammenarbeit zu stärken. Die Vielzahl unterschiedlicher diagnostischer und therapeutischer Ansätze zeigt, dass das Nierenzellkarzinom ein Paradigma für einen à priori interdisziplinär zu behandelnden Tumors ist und deshalb die therapeutisch relevanten Entscheidungen in interdisziplinär besetzten Tumorboards zu treffen sind. Für die Bearbeitung der Thematik war es freundlicherweise möglich, Experten zu gewinnen, deren aktuelle Analyse in den nachfolgenden Beiträgen abgedruckt ist.

\section{( Die MDCT gilt als das bildgebende Verfahren der ersten Wahl}

Vor allem die Multidetektorcomputertomographie (MDCT) gilt als das bildgebende Verfahren der ersten Wahl. Mit ihr lassen sich alle für eine Therapieplanung notwendigen Informationen über Lokalisation und Größe, Verhältnis des Tumors zu den Gefäßen und Nachbarstrukturen sowie zur Diagnostik von Lymphknoten und Fernmetastasen gewinnen. Vergleichbar gut ist die Magnetresonanztomographie zum lokalen Staging des Nierenzellkarzinoms. Das Fernmetastasenstaging ist eine Domäne des MDCT (U. Kramer).
Bei der Pathologie beruht die aktuelle Klassifikation der Nierentumoren auf spezifischen molekularen Veränderungen und charakteristischen biologischen Eigenschaften. Dadurch wurden in den letzten Jahren neue Tumortypen identifiziert, deren Kenntnis erheblichen Einfluss auf die Auswahl der Behandlungsstrategien hat. Unverändert ist die pathologisch anatomische Aufarbeitung von Nephrektomiepräparaten unter besonderer Berücksichtigung des Nierenhilus die conditio sine qua non zu einer korrekten TNM-Klassifikation (H. Moch).

Die Langzeitmorbidität nach Tumornephrektomie wie z. B. die Entwicklung einer Niereninsuffizienz und die damit erhöhte kardiovaskuläre Sterblichkeit haben in den letzten Jahren dazu geführt, dass die organerhaltenden chirurgischen Verfahren bei kleinen unilateralen Tumoren bei gesunder Gegenniere zum Goldstandard der Therapie geworden sind. Von besonderer Bedeutung ist, dass durch sorgfältige Selektion wahrscheinlich auch bestimmte Patienten mit unilateralem Nierentumor $>4 \mathrm{~cm}$ zukünftig organerhaltend operiert werden können (F.C. Roos).

\section{( Die Bedeutung der Indikation zur adjuvanten Systemtherapie ist unklar}

Wesentlich unklarer sind dagegen der Wert und die Bedeutung der Indikation zur adjuvanten Systemtherapie. Während derzeit keine Standardempfehlungen ge- geben werden können, lassen eine Vielzahl randomisierter Phase-II- und Phase-III-Studien (gerade auch zielgerichteter Therapien) hoffen, dass sich in den nächsten 2 bis 3 Jahren wesentliche neue Erkenntnisse ergeben werden (M. Autenrieth).

Anders stellt sich die Lage bei metastasiertem Nierenzellkarzinom dar. Hier gibt es Daten, die die zielgerichtete Therapie z. B. mit Bevacizumab, Sunitinib, Sorafenib und Axitinib in der Erst- und Zeitlinientherapie des metastasierten Nierenzellkarzinoms zur Standardtherapie oder zu einer in Einzelfällen durchzuführenden Therapie haben werden lassen. Dasselbe gilt bei selektionierten Fällen auch für die mTOR-Inhibitoren Temsirolimus und Everolimus bei genau definierten Subgruppen von Patienten. Gegenstand laufender Untersuchungen ist die optimierte sequenzielle Therapie dieser verschiedenen zielgerichteten Substanzen, sodass hierzu noch keine endgültigen Empfehlungen gegeben werden können (M. Schenck und L.-O. Mügge).

Standard sind die modernen strahlentherapeutischen Techniken in der Therapie von Lokalrezidiven und Metastasen des Nierenzellkarzinoms. Neue Entwicklungen deuten hierbei darauf hin, dass auch die Sterilisation einzelner Knochenmetastasen oder Weichteilmetastasen mit hohen Einzeldosen z. B bei einer stereotaktischen Bestrahlung den Krankheitsverlauf günstig beeinflussen können. Standardindikationen sind die schmerzhaften Metastasen wie auch die multi- 
plen Hirnmetastasen. Mit der stereotaktischen Konvergenzbestrahlung einzelner Hirnmetastasen, sei es im Sinne eines Boostes mit 10 Gy nach Ganzhirnbestrahlung oder mit Einzeitdosen von 20 Gy bei einzelnen Metastasen, ist es möglich, den Tumor zu sterilisieren. Neue strahlentherapeutische Techniken wie die intensitätsmodulierte Strahlentherapie, auch in Form der Tomotherapie, der V-MAT-Bestrahlung oder der Rapid-Arc-Bestrahlung haben dazu geführt, dass die Strahlzeit während der Intensitätsmodulation deutlich verkürzt werden kann, was die Präzision der Therapie verbessert. Auch der Einsatz der bildgestützten Strahlentherapie (IGRT) z. B. mit Cone-Beam-CT, integriert in einem Linearbeschleuniger, haben durch höhere Präzision neue Möglichkeiten eröffnet (D. Bottke).

Alle diese verschiedenen therapeutischen Ansätze zeigen, dass das Nierenzellkarzinom als Paradebeispiel einer interdisziplinär zu behandelnden Tumorintität in einem Tumorboard diskutiert und dann die Entscheidung zur multimodalen Therapie getroffen werden sollte.

Wir wünschen Ihnen eine interessante Lektüre zum Schwerpunkt Nierenzellkarzinom.

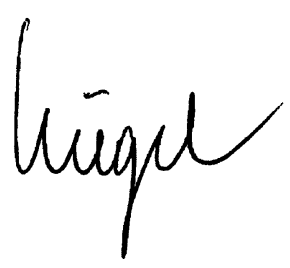

T. Wiegel

Für die Herausgeber des Schwerpunkthefts

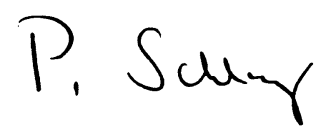

P.M. Schlag

Für die Herausgeber

\section{Korrespondenzadresse Prof. Dr. T. Wiegel}

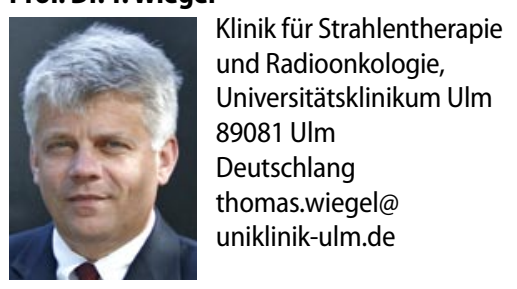

Neuer internationaler, berufsbegleitender Studiengang Master Online @dvanced Oncology

Ein umfassendes Curriculum für die onkologische Weiterbildung Die weltweite Zunahme onkologischer Erkrankungen stellt erhebliche Anforderungen an Forschung und Lehre zur Verbesserung der Versorgung krebskranker Patienten dar. Die Etablierung von Comprehensive Cancer Centers mit Hilfe der Deutschen Krebshilfe e. V. trägt wesentlich dazu bei. Onkologen sind hier in besonderem Maße gefordert, sich stetig fortzubilden, da sie in interdisziplinärer Zusammenarbeit Therapiekonzepte erarbeiten und steuern. Eine umfassende onkologische Ausbildung wird im Curriculum der ESMO/ASCO Task Force skizziert. Diese fordert neben der Vermittlung krankheitsspezifischer Leitlinien zusätzliche Kompetenzen in der Grundlagenforschung, der klinischen Forschung, der Bioethik, der Psychoonkologie sowie in rechtlichen und wirtschaftlichen Aspekten der Tumorbehandlung. Überwiegend kosten- und zeitintensive Präsenzveranstaltungen werden für die Weiterbildung angeboten. Dass die Nachfrage für ein berufsbegleitendes Programm mit flexiblem Zeitmanagement vorhanden ist, erbrachte eine Fragebogenaktion, die das ICAS, International Center for Advanced Studies in Health Sciences and Services, in Zusammenarbeit mit der Medizinischen Fakultät der Universität UIm und dem Comprehensive Cancer Center Ulm durchgeführt hat. Defizite in der Ausbildung sahen Onkologen vor allem im Bereich Management, klinischer Forschung sowie im Bereich spezielle Onkologie. Daraufhin entwickelte das ICAS den internationalen Studiengang Master Online @ dvanced Oncology. Inhaltlich orientiert dieser sich an den Vorgaben der ESMO/ASCO Task Force. Die Module interdisziplinäre Onkologie, klinische Forschung, spezielle Therapien und Management werden online in Vorlesungen und Kasuistiken bearbeitet. Präsenzseminare und zusätzliche Online-Konferenzen mit Experten werden durch Reviews und aktuelle Publikationen ergänzt. Die Zusammenarbeit der Studierenden soll zu einem internationalen, onkologischen Netzwerk führen und den Aufbau weiterer Tumorzentren voran treiben. Nach vier Semestern erwirbt man den Titel Master of Science. Zusätzlich können das Prüfarztzertifikat, das ESMO-Examen sowie EACCME-Punkte erworben werden. Voraussetzung zur Teilnahme sind der Abschluss eines humanmedizinischen oder naturwissenschaftlichen Studiums sowie englische Sprachkenntnisse. Berufspraxis auf dem Gebiet der Onkologie ist Voraussetzung. Der erste Studiengang wird zum Wintersemester 2010 starten. Der Bewerbungsschluss ist der 15. Mai 2010.

Weitere Informationen unter www.masteroncology.de. 